\title{
«Et havrà Barcellona il suo poeta». Benet Garret, il Cariteo
}

\author{
Enrico Fenzi
}

\begin{abstract}
Il saggio considera lo sfondo storico e politico contro il quale va collocata la vita (1450 circa - 1514 circa) e l'opera del poeta di origine barcellonese Benet Garret, detto Cariteo, il quale fu constantemente fedele ai suoi re. Tutta la sua opera è segnata in profondità da questa esperienza e dà forma al vero e proprio mito della Napoli aragonese. Si esaminano inoltre le testimonianze dell'opera giunte sino a noi, per passare poi a considerare la struttura del canzioniere intitolato Endimione. L'amore per Luna e l'amore per i re aragonesi e quello per gli amici e quello per Napoli non sono, in essenza, cose diverse rispetto a quella che è, appunto, la struttura profonda del canzionere. Essa finisce per comprendere anche l'amore per la patria perduta, che constituisce l'ultimo e forse più struggente mito di Cariteo il quale, alla fine della propria vita, sogna di tornare a Barcellona per affidare alla città nella quale era nato la custodia dell'intero tesoro della sua esperienza poetica e umana.
\end{abstract}

Parole chiave: Benet Garret, Cariteo, Napoli nel '500, Endimione, Barcelona.

\begin{abstract}
The thesis considers the historical and political background to the work of the Barcelona poet Benet Garret (1450 approx - 1514 approx) alias Cariteo, who was constantly faithful to his king. His work is deeply marked by this experience and relates the truth behind the myth of Aragonese Naples. Testimonies are taken toward an examination of committee work, in order to then consider the structure of the canzoniere or songbook entitled Endimione. Love for Luna, for the Aragonese king, for friends and for Naples is essentially the same thing in relation to the deep structure of the canzoniere. It terminates by also comprehending love for the lost patria that constitutes Cariteo's ultimate and perhaps most compelling myth, and expressing his lifelong dream of returning to Barcelona in order to entrust the treasure of his poetic and human experience to the city in which he was born.
\end{abstract}

Key words: Benet Garret, Cariteo, Naples in XVIth century, Endimione, Barcelona. 
Il 13 agosto 1486 a Ponzano, negli accampamenti di Alfonso duca di Calabria, era ratificato il trattato di pace firmato due giorni prima tra il re di Napoli, Ferdinando I (Ferrante), e papa Innocenzo VIII, alleato dei baroni ribelli, in guerra dall'anno precedente contro il re. Secondo le clausole, raggiunte dopo una trattativa condotta per parte aragonese dal Pontano, allora segretario di stato, Ferrante si impegnava al perdono, e a reintegrare i ribelli nei beni e negli onori perduti. Le intenzioni del re erano però diverse. Già aveva staccato dal fronte baronale Antonello Petrucci, capo della burocrazia statale, e Francesco Coppola conte di Sarno, che teneva le fila delle più importanti imprese commerciali del regno, e aveva addirittura promesso in moglie al primogenito del Coppola, Marco, una propria nipote, figlia del duca d'Amalfi. La sera dello stesso 13 agosto nel quale si firmava la pace, a Napoli, nelle sale di Castelnuovo, una folla di invitati attendeva la celebrazione delle nozze: all'improvviso, invece, alzati i ponti levatoi e isolati i presenti, comparve il castellano, Pasquale Diaz Garlon conte di Alife, che arrestò il Petrucci con la famiglia, il Coppola con i due figli Marco e Marino; Anello Arcamone conte di Borrello e messer Giovanni Pou. Contemporaneamente, a Torre del Greco veniva arrestato il secondogenito del Petrucci, Giannantonio, mentre il primogenito, Francesco, uno dei capi della congiura, fu arrestato il giorno seguente, il 14 , a Carinola. ${ }^{1}$

In queste giornate convulse, piene di colpi di scena, un segretario minore della cancelleria, il catalano Benet Garret, diventa di colpo un importante personaggio dell'amministrazione statale e quindi della corte: dal 18 agosto, infatti, comincia a figurare nei documenti come perceptor jurium regii sigilli magni — quasi un ministro delle finanze — ufficio che sino a cinque giorni prima era stato occupato dallo stesso Petrucci. Il Garret, nato a Barcellona intorno al 1450, si era trasferito a Napoli probabilmente nel 1468, al seguito di Giovanni duca di Calabria: in ogni caso, il 27 febbraio 1471 abbiamo la prima testimonianza della sua presenza nella corte di Napoli — se proprio di lui, come pare, si tratta — in una lettera di Luigi Pulci a Lorenzo il Magnifico, nella quale ripetutamente tra gli uomini di corte è nominato un Garetto: e Garectus, Garetus, Garrecta, Garret [...], è variamente designato il nostro nei documenti del tempo. ${ }^{2}$ A ridosso della promozione politica e sociale del 1486,

1. Tutto ciò è ben noto, anche per la vasta eco che ebbe a suscitare (si veda per esempio MACHIAVELli, Discorsi III 6, 4, e, ancor prima, Benedetto CROCE, Prima del Machiavelli. Una difesa di re Ferrante I di Napoli per il violato trattato di pace del $1486 \mathrm{col}$ papa. Introduzione critica e testo inedito in lingua spagnola, Bari: Laterza, 1944: la sola Introduzione è poi nei Discorsi di varia filosofia II, Bari, Laterza, 1945, p. 200-216). Ma vedi PONTANO, De sermone II 2, 27; Camillo PORZIO, La congiura de' baroni, a cura di Ernesto PONTIERI, Napoli: Edizioni scientifiche italiane, 1958, p. 137 s.; Erasmo PERCOPO, Vita di Giovanni Pontano, Napoli: ITEA, 1938, p. 46-49; Enrico PerITO, La congiura dei baroni e il conte di Policastro, con l'edizione completa e critica dei sonetti di $G$. A. de Petruciis, Bari: Laterza, 1926, p. 12 s. Il testo del trattato, in Luigi Volpicella, Regis Ferdinandi I instructionum liber, Napoli: Pierro, 1916, p. 199-210; Pietro FEDELE, "La pace del 1486 tra Ferdinando d'Aragona ed Innocenzo VIII", Archivio storico per le province napoletane XXX, 1906.

2. Seguo Giovanni PARENTI nel dare il nome secondo la forma catalana moderna (Benet Garret detto il Cariteo. Profilo di un poeta, Firenze: Olschki, 1993, p. 8 n. 2). Nel sonetto CCVII 
il Garret aveva però già goduto di una parallela promozione letteraria, perché è attorno alla metà degli anni ' 80 ch' egli aveva cominciato a figurare stabilmente tra gli intellettuali e i poeti della corte aragonese, e tra i pontaniani in ispecie. Lo mostra il Sannazzaro, sia nell' Elegia I 11, In maledicos detractores, mandata da Gabriele Altilio al Garret nel 1485, ove egli figura appunto tra i pontaniani, sia nella seconda prosa dell'Arcadia nella quale ne è sottolineata l'origine spagnola (che doveva essere ancora sentita come un elemento capace di definire il personaggio): e con ogni probabilità proprio allora e proprio dal Sannazaro gli è imposto il nome poetico di Cariteo, «alunno delle Grazie», che il Garret farà suo. ${ }^{3}$ Anni dopo, con il nome di Barcinio ('barcellonese') egli ricomparirà nell'Arcadia come interlocutore, insieme al Summonte, dell'egloga XII, e lo stesso Summonte, dedicando l'Arcadia nella stampa napoletana del 1504 al cardinale Luigi d'Aragona, ricorderà la parte avuta dal Garret nell'iniziativa: ma a questo punto la sua fama letteraria è ampiamente consolidata, e sanzionata da una ormai fitta serie di rapporti e omaggi, tra i quali spiccano quelli dello stesso Pontano che gli dedica il dialogo De splendore, lo ricorda nell'Antonius e nell'Asinus e ne fa un importante interlocutore (siamo nel 1501) dell'Aegidius. Ma non è il caso di inseguire qui tutte le testimonianze che altri ha lasciato di Cariteo (lo chiameremo così, d'ora in avanti). ${ }^{4}$ Accontentiamoci invece di osservare come la sua fama di poeta e letterato abbia cominciato ad affermarsi negli anni stessi nei quali egli entrava nella ristretta cerchia dei più alti funzionari della monarchia aragonese, quasi a indicarci, da sùbito, quella sovrapposizione e addirittura immedesimazione dei due piani che probabilmente costituisce il tratto più caratteristico e davvero unificante della sua inte-

dell'Endimione, Augustin mio, 5-6, scritto in Roma tra il 1501 e il 1503, Cariteo scrive che dopo la nascita e la giovinezza barcellonese: «Napol mi tenne poi nel bel ricetto / sette lustri». La testimonianza del Pulci, che mi pare sin qui sfuggita agli studiosi, in Domenico De RoBERTIS (a cura di) Morgante e lettere, Firenze: Sansoni, 1962, lett. XVII, p. 963-965. Cito le opere del Cariteo da: Erasmo PèrCOPO (a cura di), Le rime del Chariteo, Napoli: Tip. dell'Accademia delle Scienze, 1892, Parte seconda (la Parte prima è occupata dalla tutt'ora fondamentale Introduzione del Pèrcopo, p. XI-CCCI). Come già Parenti e la Barbiellini Amidei (vedi sotto, n. 4), ne seguo anche la numerazione, distinta per "generi metrici», nonostante la sua scomodità.

3. Eleg. I 11, 37-38: "Quin et rite suos Genio Chariteus honores / praebeat, et festas concinat ante dapes» (S., Poemata [...], Padova, Comino, 1751, p. 95); Arcadia, pr. II 8 (ed. ERSPAMER): «io ho un bastone di noderoso mirto, le cui extremità son tutte ornate di forbito piombo $\mathrm{e}$ ne la sua cima è intagliata, per man di Cariteo, bifolco venuto da la fruttifera Ispagna, una testa di ariete [...]». Egli è anche ricordato in una lettera del Galateo a Ermolao Barbaro che risale al 1481-1482, ma sembra che l'elenco dei pontaniani, tra i quali appunto il Garret, sia frutto di un rimaneggiamento posteriore (GALATEO, Epistole, a cura di Antonio ALTAMURA, Lecce: Centro di Studi salentini, 1959, p. 85-96). E sarà quasi certamente più tardo, verso gli anni '90 e non del 1482-1484, come pensa il Pèrcopo, Intr. p. CXCVI n. 2, il sonetto XCI, Marchese, ad cui natura, nel quale il Garret chiede ad Alfonso d'Avalos notizie di Andrea Matteo Acquaviva, Gabriele Altilio e Sannazaro.

4. Assai ricche sono le indicazioni del Pèrcopo, Intr. p. CXCIV s., ora rimesse a fuoco e arricchite nell'importante monografia di Beatrice BARBIELlini AMIDEI, Alla Luna. Saggio sulla poesia del Cariteo, Firenze: La Nuova Italia, 1999, passim, alla quale farò spesso implicito riferimento. 
ra esperienza. Più di qualsiasi altro — anche più del Sannazaro - a lui conviene infatti la qualifica di "poeta aragonese», per l'ininterrotta fedeltà a quella Napoli e a quei re, e insomma a quella stagione chiusa dal crollo della dinastia, nel 1501, che egli ha vissuto immerso nella luce di un ideale di regalità - quella dell'unica e vera «razza de li re» — che la sconfitta finirà per sublimare e collocare in una prospettiva trascendente: un ideale che rifulge nella storia, ma che alla storia non appartiene, e anzi su di essa trionfa. Da questo punto di vista vita e opere, in lui, vanno di pari passo, in un intreccio che si fa via via più forte, e che toccherà alle composizioni più tarde di esaltare nelle forme definitive di un destino vissuto con forte e consapevole coerenza. ${ }^{5}$

Naturalmente, ciò non significa che non ci siano vari settori della sua attività di letterato dotati di una loro autonomia, a cominciare dalle prime liriche d'amore per la bella Luna, databili con qualche approssimazione attorno agli anni ' 80 . Un capitolo affatto speciale della sua fama anche presso le corti dell'Italia del nord è per esempio affidato all'influenza determinante esercitata nei primi anni ' 90 come autore di strambotti (e come cantore e musico in proprio) su Serafino Aquilano, ${ }^{6}$ mentre, più tardi, e su tutt'altro versante, è da sottolineare l'importante l'amicizia con il cardinale, umanista e riformatore Egidio da Viterbo (1469-1532), che fu anche a Napoli e al quale il Pontano dedicò l'Aegidius, nel 1501. Di Cariteo ci resta una lettera in latino al cardinale, forse scritta durante l'esilio romano (in Percopo, II p. 463-464: gli manda le opere di Esiodo e Teocrito) che presuppone un rapporto più ampio, mentre nel dialogo stesso del Pontano compare, come s'è detto, anche Cariteo, che in un monologo tratta della creazione e della redenzione dell'uomo mediante il verbo divino, dichiara di considerarsi seguace di Ermete Trismegisto e di avere per le mani un libro che verosimilmente è il Pimander, diffusissima raccolta di testi ermetici tradotta dal Ficino per Cosimo nel 1463, e stampata a Trevi-

5. È forse opportuno richiamare brevemente alcuni dati della biografia del poeta. Anche con Alfonso II, nel 1494-1495, egli conserva la carica di "percettore». Quando poi il re abdica, il 23 gennaio, il nuovo e giovane sovrano, Ferdinando II, Ferrandino, sùbito travolto dalla rovina del regno per opera delle truppe francesi di Carlo VIII, già il 21 febbraio si imbarca per Ischia con lo zio, don Federigo, con la vedova e con la figlia di Ferrante, «seguitato da pochissimi de' suoi» (Guicciardini). Tra questi è il fedelissimo Cariteo, nominato in quella circostanza anche segretario di stato al posto del Pontano, che era rimasto in Napoli. Al fianco di Ferrandino egli fu poi in Sicilia, in Calabria e infine a Napoli, ove rientrò trionfalmente il 7 luglio cavalcando a fianco del re, con Alfonso d'Avalos. Ma l'amatissimo Ferrandino muore presto, il 7 ottobre 1496, e successore è lo zio, Federico principe di Altamura, che aumenta e rende vitalizia la rendita del Cariteo, che però esce dall'attività politica. Nel 1501, prima che le truppe di Luigi XII entrino in città (4 agosto), Cariteo si rifugia a Roma, sino al maggio 1503, quando a Napoli prende il potere Gonzalo di Cordova in nome di Ferdinando il Cattolico, sì che da regno autonomo Napoli diventa provincia spagnola. Tornato, Cariteo è nominato governatore di Nola: di più non si sa, e tutta la seconda parte della sua vita, sino alla morte, avvenuta probabilmente intorno al 1514, è assai poco documentata.

6. Per le indicazioni in merito, rinvio alla recentissima edizione di Serafino AquiLANo, Strambotti, a cura di Antonio Rossi, Parma, Fondazione Pietro Bembo-Guanda, 2002, in part. p. XVII s. 
so nel 1471. Ma un posto storicamente importante ha pure la sua accertata competenza nei confronti della lirica provenzale: negli anni romani aveva avuto rapporti con Angelo Colocci (a lui è diretto il son. CLXXIX), che dopo la sua morte, per tramite del Summonte, acquisterà dalla vedova il «libro dei poeti limosini», cioè l'attuale canzoniere $M$, Parigino fr. $12474 .^{7}$ Musico, strambottista, lirico, filosofo, erudito [...]: come si vede, lo spettro è vario, e in alcune direzioni da esplorare meglio (soprattutto per il coté speculativo, importante per i tardi poemi in terzine).$^{8}$ Resta in ogni caso vero che l'orizzonte che tutto comprende e in qualche modo sovradetermina le applicazioni particolari è quello di una pervasiva nozione di regalità, e diciamo pure di una mai smentita "fede» monarchica, che si traduce, concretamente, nell'orientare la propria esperienza di vita e di cultura secondo un arco che coincide perfettamente con quello percorso dalla dinastia aragonese negli ultimi decenni del ' 400 . Al proposito, richiamiamo assai brevemente qualche elemento che caratterizza lo sfondo contro il quale Cariteo si colloca.

Alfonso il Magnanimo morendo nel 1458 lasciava in eredità al figlio naturale Ferrante un problema politico di decisiva importanza: il consolidamento del potere regio sopra quella coalizione di forze anarchiche e anti-statali rappresentate dal baronaggio meridionale. Preso da altre immediate cure Alfonso aveva proceduto a questo riguardo con una condotta dilatoria ed equivoca, ché una linea oggettivamente statale e anti-baronale (potenziamento del ruolo dei giustizieri del regno, ritorno alla pratica del regio assenso, riforma tributaria [...]), era di fatto contraddetta da ripetuti grossi cedimenti verso i feudatari più potenti che il re, impegnato in una politica di potenza verso l'esterno, non poteva permettersi di avere nemici. ${ }^{9}$ E Ferrante ebbe infatti a scontare sùbito

7. Si tratta di un manoscritto di origine italiana (Avalle), e forse più precisamente lombardo (Folena), ornato di miniature, sulle quali vedi Maria L. MENEGHETTI, Il pubblico dei trovatori, Torino: Einaudi, 1992, p. 264-265 (ma per la bibliografia su M rimando alla BARBIELLINI AMIDEI, Alla Luna, cit., p. 184-185 nota 173). Tra l'altro sia Colocci che Summonte credevano che Cariteo avesse anche eseguito alcune traduzioni da Folchetto da Marsiglia, ma la notizia non era esatta, perché presso la sua biblioteca esisteva solo un quadernetto con testi provenzali di Arnaldo Daniello e Folchetto: per il Colocci, allora, il Summonte fece tradurre questi testi dal nipote di Cariteo, Bartolomeo Casassages (la lettera del Summonte al Colocci in Percopo I, Documenti, p. CCXCIII-CCXCVI, e in Santorre DebenedeTti, Gli studi provenzali in Italia nel Cinquecento, Torino: Loescher, 1911, p. 257-259).

8. Ma si vedano le ricche indicazioni della BARBiellini AMideI, Alla Luna, cit., p. 47 s.

9. Si veda, oltre l'utile panorama di Giuseppe GALASSO, «La feudalità napoletana nel secolo XVI», in Clio, I, 1965, p. 535 s.; Pietro GENTILE, "Lo stato napoletano sotto Alfonso I d'Aragona», in Archivio storico per le province napoletane, n. s., XXIII, 1937, p. 1-56, e XXIV, 1938, p. 1-56; Raffaele COLAPIETRA, "Gli aspetti interni della crisi della monarchia aragonese», in Archivio storico italiano, CXIX, 1961, p. 163-199 (su Alfonso in particolare, p. 168-171, nelle quali sono motivati e ribaditi i giudizi limitativi sulla sua politica interna). Per il tradizionale rimprovero rivolto al re per i privilegi riconosciuti ai baroni, vedi l'Istoria civile del Giannone, III, l. XXVI, cc. 5-7; la Storia delle Repubbliche italiane del Sismondi, LXXVII-LXXVIII, e i cenni riassuntivi di Benedetto Croce, La Spagna nella vita italiana durante la Rinascenza, Bari: Laterza, 1917 , p. 42 s. Ma per quanto si dice qui, va tenuto presente l'ampio panorama offerto dalla Storia di Napoli, nel vol. IV, del 1974, t. I (i saggi di Ernesto PONTIERI, p. 1-230; Guido D’AGOSTINO, p. 231-313) e t. II (il saggio di Mario SANTORO, p. 315-498). 
le contraddizioni della politica paterna affrontando una durissima guerra civile, la "prima congiura dei baroni» capeggiata da Giovanni Antonio Orsini principe di Taranto, ${ }^{10}$ dalla quale uscì vittorioso solo dopo aver sconfitto il 18 agosto 1462 a Troia di Puglia il pretendente angioino duca Giovanni e Jacopo Piccinino. Sin dai suoi primi atti, in ogni caso, egli aveva impresso una direzione più decisamente anti-baronale alla sua politica, intesa a favorire lo sviluppo di nuove forze cittadine naturalmente portate a identificare la propria sicurezza con quella di una solida e ben difesa monarchia, ${ }^{11}$ avvalendosi in particolare dell'opera di Diomede Carafa conte di Maddaloni, l'autore del De regis et boni principis officio, il quale, di là dalla riforma del bilancio statale, incoraggiava una politica di sgravi fiscali verso i contadini, sollevandoli almeno dagli abusi baronali più recenti, contrastati con il ricorso alle antiche consuetudini relative alle "terre comuni» e agli usi civici. ${ }^{12}$ In questo senso aveva poi un risvolto immediatamente antibaronale il riconoscimento regio accordato ai comuni e alle università dei cittadini, con la conseguente formazione di una legislazione statutaria comunale che in embrione prefigurava una organizzazione del regno diversa e alternativa a quella feudale. ${ }^{13}$ Questa linea ebbe, secondo Colapietra, il suo migliore interprete in Alfonso duca di Calabria, al quale andreb-

10. Sui suoi vastissimi possedimenti, vedi Benedetto Croce, Aneddoti di varia letteratura, Bari: Laterza, 1953, I, p. 77-79; Pietro GenTILE, Lo stato napoletano, cit., XXIII, 1937, p. 11 e note.

11. Vale forse la pena di ricordare come s'esprimeva Machiavelli, Discorsi I 55: «Questi tali [i nobili] sono perniziosi in ogni republica ed in ogni provincia; ma più perniziosi sono quelli che oltre alle predette fortune comandano a castella, ed hanno sudditi che ubbidiscono a loro. Di queste due spezie di uomini ne sono pieni il regno di Napoli, Terre di Roma, la Romagna e la Lombardia. Di qui nasce che in quelle provincie non è mai surta alcuna republica né alcuno vivere politico, perché tali generazioni di uomini sono al tutto inimici d'ogni civiltà. Ed a volere in provincie fatte in simil modo introdurre una repiublica non sarebbe possibile: ma a volerle riordinare, se alcuno ne fusse arbitro, non arebbe altra via che farvi uno regno. La ragione è questa, che dove è tanto la materia corrotta che le leggi non bastano a frenarla, vi bisogna ordinare insieme con quelle maggior forza; la quale è una mano regia che con la potenza assoluta ed eccessiva ponga freno alla eccessiva ambizione e corruttela de' potenti». E vedi, sulla sicurezza che il re può offrire al popolo, ibid. c. 16. Nelle Istorie fiorentine VII 7, si parla della politica astutamente repressiva di Ferrante nei confronti dei baroni.

12. Giovanni CASSANDRO, Storia delle terre comuni e degli usi civici nell'Italia meridionale, Bari: Laterza, 1943, soprattutto p. $216 \mathrm{~s}$. E vedi in generale, per la politica economica di Ferrante, la sintesi di Piero PIERI, Il Rinascimento e la crisi militare italiana, Torino: Einaudi, 1952, p. 91 s. (e qualche cenno al proposito in Armando SAPORI, Studi di storia economica, Firenze: Sansoni, 1955, I, p. 472 e note). Per Diomede Carafa, si veda Benedetto CroCE, Aneddoti, cit., I, p. 84-94; Tommaso PERSICO, Diomede Carafa, uomo di Stato e scrittore del secolo $X V$, Napoli: Pierro, 1899 (con la voce di Franca PETRUCCI nel DBI, 19, p. 524-530).

13. Vedi i cenni di Giuseppe Galasso, La feudalità, cit., p. 537-538, e soprattutto F. GALASSO, "La "dottrina degli statuti" per l'Italia meridionale», in Rivista di storia del diritto italiano, I, 1928, p. 492 s., con vari esempi per gli anni in questione di Statuti elaborati dalle universitates civium, sulle quali ancora Giovanni CASSANDRO, Storia delle terre comuni, cit., p. 202 s. E, dello stesso, Lineamenti di storia del diritto pubblico nel Regno di Sicilia citra Farum sotto gli Aragonesi, Bari: Laterza, 1934: ma è ora fondamentale Raffaele COLAPIETRA, Gli aspetti, cit., p. 172 s. 
be personalmente riconosciuto il merito di aver cercato di impostare «un disegno organico e coerente valido a contemperare le esigenze politiche dell'accentramento assolutistico [...] e del gettito fiscale con l'incremento conferito alle masse della piccola borghesia e delle corporazioni artigiane, non solo come contraltare alla feudalità campagnola, ma anche per limitare le tendenze egemoniche e particolaristiche dell'alta borghesia oligarchica [...]». Occorre tuttavia dire che questo quadro guarda più alle intenzioni che alla realtà: gli ostacoli che s'opponevano a un simile programma erano enormi: un dissanguante cumulo di impegni esterni manteneva l'amministrazione aragonese al limite di un perenne fallimento, e la spingeva a una disastrosa caccia ai soldi nella quale, di fatto, erano rinnegate scelte politiche di fondo, mentre la povera economia rurale e il difficile mondo delle intrapprese economiche erano soffocati dal parassitario monopolio mercantile fiorentino e veneziano. Di più, l'incredibile fragilità militare, conseguenza diretta delle disastrose condizioni sociali ed economiche, esponeva il regno a pericolosi contraccolpi, come, nello sbalordimento generale (e nella malcelata soddisfazione degli altri, in primis $\mathrm{i}$ veneziani), rivelò la presa di Otranto da parte dei Turchi nel $1480 .{ }^{14}$ A tutto ciò s'aggiunga l'ostilità della grande borghesia oligarchica, ben rappresentata dal Coppola, che non poteva non essere toccata nei propri interessi da una politica quale quella perseguita, pur con incertezze, da Alfonso il Magnanimo e da Ferrante, e che di fatto s'alleò con i baroni ribelli. ${ }^{15}$ Questo spiega come, nonostante la vittoria sui baroni e l'indubbia efficacia della repressione, e nonostante il rinvigorirsi, attorno al 1490, della linea politica di appoggio alle autonomie cittadine e di stimolo a nuove attività imprenditoriali, l'invasione francese del 1494 dilagasse in un paese nel quale davvero poche cose avevano avuto modo di maturare: nel vecchio regno, insomma, nel quale ancora una volta i baroni erano riusciti a ricomporre la loro forza eversiva. E spiega, infine, come l'ultimo dei re aragonesi, Federico, posto sul trono dai baroni angioini che pare avessero fatto abortire un tentativo di parte popolare per far succedere a Ferrandino la vedova Giovanna, abbia rinnegato rapidamente la tradizionale politica aragonese di contenimento del potere baronale e di alleanza con i ceti borghesi, e in un regno minato dalla consapevolezza della prossima catastrofe si sia forzatamente adattato alla pura e semplice alleanza con le grandi famiglie baronali, reintegrate nei loro privilegi.

14. Vedi Pietro EGIDI, «La politica del Regno di Napoli negli ultimi mesi del 1480», in Archivio storico per le province napoletane, XXXV, 1910, p. 697-773, ove è ben documentata la povertà e la debolezza di Ferrante, e lo stupore di fronte a ciò del papa e dei principi italiani. Parte dei documenti citati dall'Egidi sono in Cesare FOUCARD, «Fonti di storia napoletana nell'Archivio di Stato di Modena: Otranto nel 1480 e 1481", in Archivio storico per le province napoletane, VI, 1881, p. 165 s. e p. 611 s. E ancora, per le disastrose condizioni delle finanze regie, Piero PIERI, Il Rinascimento, cit., p. 94-95.

15. Analizza i difficili rapporti Coppola - Ferrante - Alfonso, Raffaele ColapiETra, Gli aspetti, cit., p. 186-187. Più in generale, è poi importante dello stesso COLAPIETRA l'ampio saggio "Aquila e l'Abruzzo nell'età aragonese», in Rivista storica del mezzogiorno, marzo-aprile 1966, p. 61-166. 
Qualcosa ad ogni modo era stato costruito (e torniamo con ciò a Cariteo): una amministrazione statale più moderna, alla quale era addetta una classe cittadina di funzionari regi e giuristi che la corona aveva via via aggregato alla nobiltà di Seggio, chiusa verso la grande aristocrazia feudale a sua volta largamente disimpegnata e avversa ad assumersi responsabilità negli affari municipali. ${ }^{16}$ Questa classe, che aveva ormai oggettivamente una funzione anti-feudale e anti-baronale, tendeva a uscire dal vecchio schema della famiglia del re, e a inserirsi, invece, in un complesso di attribuzioni e attività proprie di una struttura statale. Ed è solo e precisamente in questo quadro che si può capire come un peso affatto nuovo assumesse Napoli quale città-capitale, "preciso ubi consistam geografico e sociale alla classe amministrativa», ${ }^{17}$ e come ne rinascesse in forme nuove il «mito» di Napoli, affidato alle cure degli intellettuali ai quali toccava di mostrarne l'intimo legame, quasi di consustanzialità, con quello della casa regnante: come spicca nel caso emblematico dello spagnolo poeta di corte Diego del Castillo, autore di una lunga visione sulla morte di Alfonso il Magnanimo, ma anche, nella stessa occasione, a completare il dittico, di una appassionata elegia funeraria che «Partenope la fulgente» (e sarà poi Cariteo a fare del fulgore uno dei tratti fondanti del suo mito monarchico) rivolge alla vedova, la regina Maria. ${ }^{18}$

A questo proposito è assai interessante osservare come anche i progetti urbanistici del duca di Calabria derivino dalla linea politica sua e del padre, tesa al consolidamento del potere regio: il duca, così come ricordava il Summonte molti anni dopo, avrebbe voluto una Napoli nuova, funzionale, degna capitale di un forte stato monarchico, fatta di strade larghe e diritte dalle quali fossero spazzati via i segni del particolarsmo medievale, le enclaves dei vecchi ceti dominanti. Unico sarebbe emerso il Pantheon della dinastia regnante, vicino al quale sarebbero stati gli edifici riservati ai supremi organi amministrativi e burocratici dello stato. ${ }^{19}$ Certo, la monarchia giuse solo per gradi e con varie

16. Vedi Martino BerEngo, L'Europa delle città. Il volto della società urbana europea tra Medioevo ed Età moderna, Torino: Einaudi, 1999, p. 319 s.

17. Sono parole di Giuseppe Galasso, La feudalità, cit., p. 542.

18. Rimando per ciò all'analisi dell'elegia e all'ampia bibliografia in merito di Lourdes SIMÒ Goberna, Una elegia epistolar a la muerte del Magnanimo (1998), che per ora sono riuscito a leggere in Internet, all'indirizzo: http://parnaseo.uv.es/Lemir/Revista/Revista3/Lourdes/Elegia.htm.

19. Ecco parte di quel che scrive il Summonte nella lettera al Michiel del 1524, ricordando i progetti di Alfonso II: «Ad tempi nostri lo signor re Alfonso secondo di felicissima memoria fo tanto dedito alla fabrica e cupido di far cose grandi che, se la iniqua fortuna non lo avesse deturbato così presto dal suo solio, senza dubbio averia ornata questa città. Erat illi in animo [...] extendere ad linea recta tucte le strade maestre, da muro a muro, della città, tolti via tutti i portichi, cantoni e gibbi ineguali, e così per traverso extendere pure ad directura tucti li vichi da capo al capo della città [...] Oltra a ciò, volea ancora edificare un templo sumptuosissimo e ivi ponere le ossa di tutta la progenie Aragonia delli signori ch'erano morti qua, e un palazzo grande vicino al Castello Novo nella piazza della Coronata, nella quale volea per diverse stanzie collocare tutti li tribunali», ecc. La lettera fu ritrovata da Fausto NiCOLINI, e da lui pubblicata, prima in "Napoli nobilissima» del 1922 e poi in volume: L'arte napoletana del Rinascimento e la lettera di Pietro Summonte a Marcantonio Michiel, 
incertezze a una consapevolezza anche ideologica di questo tipo, e fu soprattutto la congiura dei baroni del 1484-1486 a determinare una svolta più decisa. La reazione baronale alla lenta pratica aragonese di contenimento e di erosione della struttura feudale del regno fu infatti seguita dalla feroce repressione regia, che da quel 13 agosto 1486 s'esercitò poi per anni e fece cadere molte teste. $\mathrm{E}$, da sùbito, pose alla corte un problema di uomini. Per quanto riguardava l'alta nobiltà, per esempio, su sette grandi ufficiali del Regno due soli restarono fedeli al re: il gran giustiziere Antonio Piccolomini duca d'Amalfi e il gran protonotario Onorato Caetani conte di Fondi; ambiguo fu l'atteggiamento del gran cancelliere Jacopo Caracciolo conte di Brienza, mentre si ribellarono gli altri: il gran connestabile Pirro del Balzo principe d'Altamura; il grande ammiraglio Antonello Sanseverino principe di Salerno; il gran camerlengo Gerolamo Sanseverino principe di Bisignano; il gran siniscalco Pietro di Guevara marchese del Vasto (e con i ribelli furono pure i due più importanti personaggi dell'amministrazione statale, il Petrucci e il Coppola, come abbiamo visto). Tutto questo certo ha influito sul re nel fargli compiere una scelta che contraddiceva a una pratica politica da lui mantenuta con una certa costanza, quella cioè di scegliere persone originarie del regno a ricoprire cariche pubbliche: ci si riferisce, appunto, alla nomina del barcellonese Cariteo a conservatore del regio sigillo, al posto del Petrucci. ${ }^{20}$ Già s'è accennato a quel poco che si

Napoli: Ricciardi, 1925. Il passo circa Alfonso II e i suoi progetti anche in Ottavio MoRISANI, Letteratura artistica a Napoli tra il ' 400 e il '600, Napoli: F. Fiorentino, 1958, p. 6061. Qui, p. 32 s., c'è anche un'abbondante documentazione sulle numerose imprese edilizie volute e seguite da Alfonso, quand'era ancora duca di Calabria (ora si veda anche la gradevole esposizione che è nel volume di Marcello OrEFICE, Napoli aragonese tra castelli, palazzi, vicoli e taverne, Napoli: Electa Napoli, 1999, con bibl.; ma anche Roberto PANE, Architettura e urbanistica del Rinascimento, nel citato vol. IV, t. I, della Storia di Napoli, e Jerry H. BENTLEY, Politica e cultura nella Napoli Rinascimentale. Intr. di Giuseppe GALASSO, Napoli: Guida, 1995, passim). Aggiungo che quanto qui appena si accenna circa la forte componente ideologica del «programma» monarchico degli aragonesi prende sùbito corpo con Alfonso il Magnanimo (basterebbe fermarsi, per ciò, sull'arco di Castelnuovo, del Laurana), la cui corte costituisce, tra l'altro, il vero punto focale attraverso il quale passa lo scambio culturale tra Spagna e Italia, e che lo si potrebbe immediatamente allargare, attraverso una bibliografia ormai vastissima. Qui, mi limito tuttavia a segnalare un recente volume: Gabriella ALbANESE (a cura di), Studi su Bartolomeo Facio, Pisa: ETS, 2000, nel quale sono ben colti i caratteri della storiografia ufficiale di corte, ben rappresentata nelle sue coordinate fondamentali dai Rerum gestarum Alphonsi regis libri $X$ dello stesso Facio.

20. Sulla fitta presenza di catalani alla corte di Alfonso il Magnanimo, vedi Benedetto CrocE, La Spagna nella vita italiana, cit., p. 32 s., e sul diverso indirizzo seguito poi da Ferrante, ibid., p. 56 s., più volte tradìto, come ebbe egli stesso a lamentare, dai "criati» spagnoli e catalani del padre (vedi Francesco Senatore, Pontano e la guerra di Napoli, nel vol. misc. Mario Del Treppo (a cura di), Condottieri e uomini d'arme nell'Italia del Rinascimento, Napoli: GISEM, 2001, p. 281-311). A questo proposito è interessante la "proposta» segreta fatta i primi mesi del 1445 ad Alfonso da Borso d'Este, dove si indica come motivo di grave debolezza interna del regno il favore accordato dal re ai catalani, «per la quale cossa pare che la V. Maystà non se fidi de veruno Italiano» (Cesare FouCARD, "Proposta fatta dalla Corte Estense ad Alfonso I re di Napoli (1445)» Archivio storico per le province napoletane, IV, 1897, p. 714). Lo stesso senso che possiamo attribuire alla nomina del Cariteo può esse- 
sa del Cariteo sino al momento in cui, in circostanze così delicate e in una situazione assai tesa, fu posto dal re su un alto gradino della scala burocratica. Col senno di poi, si può aggiungere che sul piano della fedeltà la scelta non fu sbagliata: il barcellonese non nobile, non ricco (così pare, se si sta a quanto scrive il Pontano dedicandogli il De splendore nel 1494: «Ac tametsi familiaris res tua domesticaque suppellex sit etiam mediocrior [...]»), in nessun modo legato alla locale nobiltà ribelle se non per il tramite di comuni interessi letterari, com'è il caso di Andrea Acquaviva e Giannantonio Petrucci, ${ }^{21}$ e semmai stretto da più vincoli proprio con i fedeli degli Aragonesi, i d'Avalos prima di tutti, e tra questi i fratelli Alfonso e Costanza, e poi con il Pontano e il Sannazzaro e gli altri colleghi negli uffici di corte, era nell'oggettiva condizione di chi doveva porre nella dinastia regnante ogni sua speranza, e identificare il suo personale destino con il destino di quella.

A costo di ripetere cose in parte già dette, è bene illustrare come ci sia giunta l'opera di Cariteo, e darne una descrizione almeno sommaria. Possediamo, prima di tutto, un manoscritto, già di proprietà di Tammaro De Marinis, passato poi per il mercato antiquario, e ora disponibile agli studiosi presso la Fondazione Anna Maria e Mariella Marocco di Torino, per la tutela del libro manoscritto e stampato. Tale codice, descritto già da Contini quand'era ancora di proprietà del De Marinis, e studiato dopo la sua ricomparsa da Beatrice Barbiellini Amidei e da Paola Morossi, che ne sta preparando l'edizione, ${ }^{22}$ corrisponde in gran parte alla prima edizione che, con il titolo già del codice di Endimion a la Luna, venne stampata a Napoli il 15 gennaio 1506 da Giovanni Antonio de Caneto. Scoperta dal Percopo l'anno stesso nel quale usciva la sua edizione, $1892^{23}$ nell'unico esemplare sino ad oggi conosciuto: Bibl. Estense, mss.

re esteso a quella del suo caro amico, e pur egli pontaniano, lo spagnolo Giovanni Pardo, che il 23 marzo 1487 è nominato segretario della regia Cancelleria. A lui il Pontano dedica il De conviventia, e ne fa un bel ritratto nel De sermone. Vedi Camillo MINIERI RICCIO, Biografie degli accademici alfonsini detti poi pontaniani dal 1442 al 1543, s.a. né l. né ed. (ma Napoli, 1880-1882), p. 12-16: cito dall'anastatica di Bologna: Forni, 1969).

21. Ma Andrea Matteo Acquaviva, inizialmente con i baroni ribelli, se ne staccò presto e consegnò i castelli, sì che alla fine del 1486 già era riammesso a corte (Camillo PORZIO, La congiura, cit., p. 41). Cariteo lo ricorda ed esalta nell'Endimione, XCI, Marchese, ad cui natura diede ingegno; nella Resposta contro li malivoli, 190-195 s., e nella Pasca, VI 98-99, mentre il Pontano gli dedicò il De magnanimitate. Su di lui, tradizionalmente lodato quale soldato e letterato insieme, vedi la voce redazionale Acquaviva d'Aragona A. M. nel DBI I, 1960 , p. 185-187. Nel 1486, dal carcere nel quale era stato rinchiuso in attesa dell'esecuzione (avverrà per decapitazione l'11 dicembre dello stesso anno), Giannantonio Petrucci, figlio di Antonello, rivolgeva a Cariteo un sonetto, Conosco contra me sì adverso fato (LXVIII: ed. Perito, cit., p. 266-267).

22. Gianfranco ConTINI, Il codice De Marinis del Cariteo, nel vol. misc. Studi di bibliografia e storia in onore di Tammaro de Marinis, Verona, Valdonega, 1964, II, p. 15-31; Giovanni PARENTI, Benet Garret, cit., p. 3-7; Beatrice BARBIELlini AMIDEI, Alla Luna, cit., p. 27-38; Paola Morossi, Il primo canzoniere di Cariteo secondo il codice Marocco, «Studi di Filologia Italiana», LVIII, 2000, p. 173-197.

23. Erasmo PÈRCOPO, "La stampa napoletana del 1506 delle Rime del Chariteo», in Giornale storico della lett. italiana, XX, 1892, p. 314-317. 
A.Z. 2. 12, contiene un prologo al cavalier Cola d'Alagno, e il canzoniere, che dal codice alla princeps passa da 61 a 65 componimenti: $41>45$ sonetti ( i nuovi portano i numeri XLIX; L; LI e LIII nell'ed. Pèrcopo); 5 canzoni; 3 madrigali; 3 ballate; 3 sestine; 6 canzoni a rimalmezzo delle quali solo la prima ha regolare divisione strofica. Dopo alcune carte bianche, è presente una compatta sezione di strambotti (33 nel codice, e 32 nell'edizione, che ne ha due in meno, Quelle vane speranze che mi diste, e Poi che ad madonna il mio cantar dispiace, e uno in più, Poi che la Luna e 'I Sol con l'altre stelle), alla quale segue una breve dedica ad Alfonso d'Avalos che precede due canzoni: una in lode di Ferrandino ancora principe di Capua, La candida vertute al cielo eguale (canz. VII nell'ordinamento finale, al quale farò sempre riferimento, di 148 versi), cui segue l'altra, Alza la testa al polo (canz. VI, v. 309), ad esaltazione della dinastia aragonese, intitolata Aragonia.

La prima edizione del canzoniere, dunque, più un'antologia che un'opera a sé, come mostra la struttura dell'insieme, riproduce "con notevole cura, un esemplare vicinissimo al nostro codice, ma posteriore o almeno un po' arricchito» (Contini, p. 18: questa vicinanza è ora confermata dall'analisi delle varianti tra il codice e la stampa fatta dalla Morossi). La possibile data di composizione delle liriche s'arresta alla fine del 1493: il codice infatti porta le armi di Ferrandino ancora principe di Capua, e dunque precede il 25 gennaio 1494, quando, salendo Alfonso II al trono, egli assunse automaticamente, in quanto diretto successore (ma a ciò per la verità era stato designato già dal 1492), il titolo di duca di Calabria: altri elementi interni alla canzone che gli è dedicata, e all'altra, Aragonia, confermano del resto questa datazione. Ciò potrebbe far pensare che l'iniziativa della stampa, nel 1506, che ripropone senza alcun aggiornamento e con pochissime variazioni quella raccolta, avvenisse per vie proprie, senza un coinvolgimento diretto dell'autore, ${ }^{24}$ il quale ne fu forse stimolato a riprendere la sua intera produzione per la successiva e completa edizione del 1509. La quale fu pubblicata a Napoli, per le cure dell'amico Pietro Summonte, nel novembre 1509 da Sigismondo Mayr: ed è quella riprodotta dal Percopo nel 1892, e sulla quale dovrà in ogni caso basarsi ogni futura edizione. Qui, il canzoniere passa da 65 a ben 247 componimenti (214 sonetti; 20 canzoni; 5 ballate; 5 sestine; 3 madrigali). Dopo sei sonetti proemiali, dal settimo è sostanzialmente riprodotto, con pochissimi spostamenti interni, il canzoniere del 1506, che occupa dunque il primo quarto, circa, del nuovo Endimione. Della prima edizione sono rifiutate le 6 canzoni a rimalmezzo e i 32 strambotti, mentre le due lunghe canzoni là isolate, oltre il canzoniere, sono ora inglobate in esso, in ordine inverso, come già s'è visto. Ad esso seguono altre opere, tutte sin lì inedite: 6 canzoni per la natività della Vergine; una can-

24. Già la Morossi scrive che «Nulla ci autorizza a considerare la princeps dell'Endimion come il prodotto della volontà del Cariteo", e che la sua testimonianza, praticamente coincidente, dal punto di vista redazionale, con quella del codice Marocco è appunto una testimonianza epigonica, «stampata verosimilmente senza controllo dell'autore» (Il primo canzoniere, cit., p. 186-187). 
zone per la natività di Cristo; una canzone in laude de la humilitate; un cantico in terzine de dispregio del mondo; il Libro de la Methamorphosi, in terzine, in tre cantiche (v. 139; 187; 245); un cantico in terzine per la morte di Inico d'Avalos marchese del Vasto, indirizzato a Costanza d'Avalos, sua sorella (v. 256); una Resposta contra li malivoli, in terzine (v. 229); il Libro intitulato Pascha, poemetto religioso in sei cantiche (v. 247; 181; 217; 157; 184; 181). A differenza dell'Endimione e con l'eccezione della Resposta (precede il settembre del 1495, perché Alfonso d'Avalos vi compare ancora in vita), tutte queste opere sono state scritte dopo il 1500.

Torniamo all'Endimione. Il passaggio dalla prima alla seconda e definitiva edizione comporta determinanti novità. La prima sta nel fatto che il canzoniere comprende ora ben 247 componimenti, contro i 65 della precedente; la seconda, che i 61 componimenti che passano da una all'altra subiscono una profonda doppia revisione, stilistica e, soprattutto, linguistica, attraverso la quale Cariteo, in parallelo con quanto faceva l'amico Sannazzaro, cerca di depurare la propria lingua e di normalizzarla, pur con qualche incertezza e contraddizione, avvicinandosi al modello toscano, a metà tra la soluzione petrarchesca e arcaica e quella del toscano di secondo Quattrocento. Non è tuttavia di questo particolare aspetto che vorrei parlare, ${ }^{25}$ ma d'altro ancora, che ha a che fare con la struttura apparente, se posso dire così, e con quella profonda del canzoniere. Prima di tutto occorre dire che il "primo" Endimione $(=A)$ presenta la situazione topica dell'amante non corrisposto come una situazione bloccata, interamente data sin dal primo sonetto e priva di svolgimento, di storia. Da questo punto di vista, mi pare fondamentale sottolineare che questa prima raccolta non è un canzoniere, ma appunto una raccolta di rime d'amore, se con canzoniere intendiamo qualcosa di diverso: essenzialmente, quella «avventura organica dell'anima», quella "cosciente costruzione psicologica [...] chiusa nell'armatura d'una storia perspicua» di cui parla così bene Contini proprio all'inizio della sua Introduzione alle rime di Dante. ${ }^{26}$ Non lo è dunque: ma bisogna andar cauti, perché per un altro verso lo è. Leggiamo il primo sonetto, nel testo di $\mathrm{A},{ }^{27}$ cosa che ci permette tra l'altro di non fermarci troppo sulla "poetica» di Cariteo, da lui stesso espressa in modo tanto eloquente quanto efficace:

25. Rimando per questo al mio saggio E. FENZI, «La lingua e lo stile del Cariteo dalla prima alla seconda edizione dell' "Endimione" ", in Studi di filologia e letteratura, I, 1970, p. 9-83 (pubblicati dall'Istituto di letteratura italiana dell'Univesità di Genova).

26. La cosa è ben notata già dalla Morossi, che scrive: «importa qui notare che una storia non c'è»; "questo canzoniere appare situazionale e non narrativo", e ancora "La situazione è data fin dal primo momento, e non subisce sviluppi significativi», ecc. (Il primo canzoniere, cit., p. 185: le parole in corsivo la Morossi le ricava da un saggio di Cesare BOzZETTI, ove sono riferite al Sannazzaro, "Note per un'edizione critica del "canzoniere" di Iacopo Sannazzaro", in Studi di filologia italiana, LV, 1997, p. 111-126 [p. 123]).

27. Ecco le varianti del testo definitivo, B: 1 sospirar e l cantar mio; 2 se l'imp. ; 3 voci; 4 f. la pietà porre in oblìo; 5 p. il fallir mio; 6 a te p. ; 7 mi; 10 disfogar; 11 occolta [...] del; 12 mezzo a. 


\begin{abstract}
Amor, se 'l suspirare e 'l cantar mio et l'importuno amaro aspro lamento, le voce triste in doloroso accento t'han fatto hor più sfrenato, hor più restio, ad te stesso perdona e al van desio come prima cagion del mal ch'io sento, però che se cantando io me lamento, tu sei quel che si lagna, e non son io. Et benché al mio cantar nessun risponde, pur canto per sfogare il duol ch'io premo ne la più occulta parte dil mio core. Io son pur come il cygno in mezo l'onde, che quando il fato il chiama al giorno extremo alzando gli occhi al ciel cantando more.
\end{abstract}

Certo non è quel sonetto proemiale, scritto per ultimo e posto per primo onde traguardare l'intera vicenda, che ci si aspetta da Voi che 'ntendendo in avanti. Eppure in qualche modo ha anch'esso un tono latamente proemiale, in quanto dichiarazione di poetica che postula l'unità — l'unica data come possibile - della vicenda d'amore appunto nel segno della sua irrisolvibile, obbligata immobilità. Cariteo per primo caratterizza con grande precisione e verità la sua poesia amorosa come un "canto del cigno», ogni volta riassuntivo, totalizzante, testamentario e, aggiungiamo ancora, proprio in quanto tale rivolto sempre all'esterno, gridato innanzi al mondo. E del resto, quale «avventura organica dell'anima» ci sarà mai da narrare, quando ciò che doveva avvenire già è avvenuto? quando non c'è da «dire» altro se non, puramente e semplicemente, la condizione d'innamorato? Che anche in ciò sia da vedere, di là dal magistero stilistico e linguistico di Petrarca, la determinante presenza di modelli classici, e tra essi in particolare quella di Properzio, mi pare indubitabile. ${ }^{28} \mathrm{Ma}$ lasciamo anche questa via, e vediamo invece come questo "canzoniere-nonpetrarchesco» (una sorta di ossimoro abbastanza intrigante [...]) sia accolto nella assai più vasta compagine del secondo Endimione, cioè nella definitiva edizione del $1509(=B)$. Qui, già abbiamo detto, dopo sei nuovi sonetti introduttivi, è ripresentata la vecchia raccolta, dal sonetto VII al LIII, al quale seguono direttamente le due canzoni politiche VI e VII, in ordine capovolto. Sono però escluse le sei rime al mezzo già incorporate nel corpus amoroso e gli strambotti, che invece occupavano una sezione a parte, prima di quelle due canzoni. Gli spostamenti interni sono pochi, mentre fa eccezione il sonetto già $\mathrm{n}$. 3, Quando l'aurora il chiaro giorno adduce, che ora si è staccato dagli altri e,

28. Che la presenza dei modelli latini, rivissuti con intima adesione, costituisca un tratto fondamentale della poesia di Cariteo e le conferisca un suo inconfondibile "tono" anche concettuale e fantastico è stato da tempo riconosciuto: per ciò, si veda in particolare, oltre a quanto si ricava dalle monografie di Parenti e della Barbiellini Amidei, Rino ConsOlo, «Il libro di Endimione: modelli classici, "inventio" ed "eloqutio" nel canzoniere del Cariteo", in Filologia e critica, III, 1978, p. 19-94; Claudia FANTI, "L'elegia properziana nella lirica amorosa del Cariteo», in Italianistica, XIV, 1985, p. 23-44. 
sottoposto a fitta revisione, è diventato il CXXIX. Solo quattro sono i componimenti nuovamente inseriti, i sonetti XVI, Da che si leva il sol da i rosei scanni, e XXXV, Quando mi tiene Amor per sua natura; la ballata III, Per saper l'hora incerta; il sonetto LII, Quando col mio periglio ardire io prendo. Mentre per gli ultimi tre non occorrono osservazioni immediate, si nota che il XVI segue a due sonetti che in $A$ erano lontani uno dall'altro (n. 11 e 40), e che ora sono riuniti per l'affinità del contenuto (il poeta in sogno vede Luna ignuda e arriva a soddisfare il suo desiderio): il nuovo sonetto completa dunque secondo una coerente linea di sviluppo tematico l'elegante colorito erotico dei due precedenti. $^{29}$

Come s'è detto, $A$ enuclea un momento alto e pieno della storia amorosa: in $B$, con il sonetto 54, che segue alla canzone in lode di Ferrandino e inaugura la parte affatto nuova del canzoniere, si torna a quel momento per riprenderne il filo, come dichiara il poeta stesso mettendo in mostra il punto di sutura tra vecchia e nuova raccolta: «Or ritornamo ai primi aspri tormenti, / a le lagrime prime, al primo ardore [...]» (v. 1-2). Da questo punto in poi cambia qualcosa, perché la "condizione» amorosa comincia a innervarsi in una storia: Cariteo infatti si preoccupa di segnare i tempi secondo i quali si articola la sua vicenda, ricostruibile per noi a partire dal suo culmine — la partenza di Luna per la Spagna - sulla quale occorre fermarsi un momento. Questa partenza è collocata al 10 ottobre 1492, perché il poeta ne ricorda il primo anniversario il 10 ottobre dell'anno successivo, come conferma l'indicazione contenuta nella canzone XV circa l'eclissi di sole che quel giorno interessò tutta l'Europa (v. 27-29 e 40-52). Altri elementi che confermano questa data non mancano: nel son. CXLVI, 9-11, si dice che Ferrante, morendo (25 gennaio 1494) lasciò Napoli senza Luna; nei sonetti CXXVII e CXXIX il poeta se ne lamenta con Ferrandino ancora principe di Capua, (siamo dunque prima del 25 gennaio 1494); nei sonetti CXXVI e CXXVIII se ne lamenta col marchese di Pescara Alfonso d'Avalos, che morì il 7 settembre 1495 [...] Il sonetto CXLV, scritto anch'esso per il primo anniversario della partenza, si apre così: "Un anno è, Luna mia, che sei partita, / e tredeci che me di me togliesti», donde risulta che la data dell'innamoramento è posta nel 1480. Di qui è possibile tornare ai tempi che Cariteo allinea in bell'ordine. Nel sonetto LIX, 5-6: «già passato è l'anno sesto / de la mia pena» (1486); nel son. LXXII, 9: "volge il septimo anno» (1487: e vedi ancora sonn. LXXXIX e CII). Nella canzone X, 24-25, diretta al Sannazaro, la quale chiude la fase della storia d'amore nella quale ancora non si parla della partenza di Luna: «passa il decimo anno, / ch'io pugno meco per fuggir d'affanno" (1490). I componimenti che sfuggono a questa progressione cronologica entro la zona che termina con la canzone al Sannazaro non sono molti e non sono amorosi (son. CXIII a Laura Sanseverino, per la morte del marito Inico d'Avalos, settembre 1503; sonn. XCI-XCII, del 1482-1484; son. CX a Costanza d'Avalos, posteriore all'aprile 1501).

29. Si vedano ancora i versi finali, 45-48, piuttosto "pontaniani», nella prima delle canzoni rifiutate, Luce de la età nostra, e i simili sonetti del Sannazaro, LXI-LXII e LXV. 
Il momento successivo della storia d'amore abbraccia in modo compatto i componimenti CXVI-canzone XI, tutti dedicati al tema doloroso della partenza di Luna per la Spagna. Nel son. CXVI la partenza è annunciata al poeta in un sogno «paventoso, oscuro e nero", mentre nella canzone XI è descritto minutamente l'imbarco di Luna e lo sparir della nave all'orizzonte.

Il terzo e ultimo momento della storia riguarda ormai solo i lamenti del poeta lontano dalla sua donna, lamenti che da un certo punto in poi vanno diradandosi per lasciare il posto a nuovi impegni politici e celebrativi. In particolare per Luna lontana sono le liriche dal son. CXXV al CXLVII, con poche eccezioni, la più notevole delle quali è rappresentata dalla canzone XIII, ad Alfonso d'Avalos, per la morte della madre. Entro questa serie, per il primo anniversario della partenza sono il son. CXLVI e la canzone XV (ma vedi anche la canzone XIV, 75-76), importante questa perché permette, come abbiamo visto, di datare con precisione la partenza stessa al 10 ottobre 1492.

A questo punto, tra il son. CXLVII e la successiva canzone XVI, scritta per la salita al trono di Alfonso II (25 gennaio 1495), ${ }^{30}$ c'è uno stacco preciso. Qui infatti Cariteo termina il suo canzoniere amoroso e si rivolge a quei temi che egli ritiene più impegnativi: la celebrazione e la difesa della dinastia aragonese nel momento in cui incombeva l'arrivo di Carlo VIII, e tutta un'epoca stava per finire. Naturalmente la poesia amorosa non sparisce di colpo e per una certa zona il canzoniere procede per brevi sezioni alternate. Qui, basti ricordare che il son. CLIV, «flebil canto d'absentia e di morte», piange insieme la lontananza di Luna e la morte del giovane re, avvenuta il 9 ottobre 1496. Per Luna ci saranno ancora cinque sonetti (CLVI; CLXII-CLXIV, e CLXXVII). In CLXIV ricorda come Luna avesse lasciato Napoli «dece anni or son, e con lor giunto un mese»: fu scritto quindi nel novembre 1502. In Roma, e perciò ancora negli anni 1502-1503, fu scritto l'ultimo sonetto per Luna, il CLXXVII, nel quale si accenna al suo ritorno dalla Spagna, che, se ci fu, rimase per quanto ci interessa senza conseguenze.

Il son. CLIV, il canto «d'absentia e di morte» del 1496, aveva in ogni modo segnato un'altra svolta entro il canzoniere: con il sonetto successivo, infatti, dedicato a Pier Giovanni Spinelli, comincia l'ultima parte che, eccettuati i cinque sonetti di cui s'è detto e la ballata $V$ sull'umiltà, è tutta composta di sonetti non d'amore diretti, dopo il 1500, a potenti e amici: Andrea di Capua, Oliviero Carafa, il Summonte, Lelio Gentile, Girolamo Carbone, Paolo Cafatino, Isabella Gonzaga, Piero Pazzi, Gianvincenzo Carafa [...] L'unica canzone in questa zona è l'ultima, la XX, che chiude il canzoniere, diretta al cardinale Carafa, scritta assai probabilmente nel 1508.

Si possono derivare, a questo punto, alcune conclusioni generali sulla composizione del canzoniere, così riassumibili: l'Endimione è composto da una

30. Su Alfonso II e sui numerosi omaggi letterari che gli sono stati indirizzati, si veda Antonia Tissoni Benvenuti, Alfonso duca di Calabria e le "Pastorale» del Boiardo, nel vol. misc. Franca Magnani (a cura di), Studi in memoria di Paola Medioli Masotti, Napoli: Loffredo, 1995, p. 47-55. 
serie di liriche d'amore disposte secondo lo sviluppo della vicenda (innamoramento-partenza-rimpianto) dal 1480 al 1493 circa. In questo stesso anno Cariteo comincia ad affrontare argomenti più ambiziosi, ai quali è richiamato dalle particolari condizioni politiche del Regno e dalla sua diretta partecipazione agli avvenimenti del 1494-1496, e questo nuovo impegno fa sì ch'egli smetta quasi del tutto d'essere poeta d'amore. Dal 1496 circa (pochi sono i componimenti riferibili a quell'anno) al 1500 c'è il vuoto, mentre l'ultima parte, assai omogenea, per una sessantina di componimenti su 247, è tutta posteriore al 1500 , e testimonia nei suoi momenti migliori una fase di ripensamento, spesso in colloquio con gli amici più cari, delle idealità e dei valori sui quali egli aveva creduto di regolare la sua vita.

Riconsideriamo le cose nell'insieme, e chiediamoci: l'Endimione (quello definitivo, s'intende) è un canzoniere d'amore? Sì e no. Le liriche d'amore, disposte secondo $\mathrm{i}$ «tempi» della vicenda, ne occupano più della metà (i sei decimi, poco più o poco meno): ma ciò che, in linea di fatto e di principio, fa davvero la differenza, è che il percorso dell'esperienza amorosa non copre l'intero percorso dell'opera. Decisivo è infatti che la parte non amorosa non sia affidata a poesie d'altro argomento variamente e magari abbondantemente mescolate alle amorose, ma sia appunto una parte successiva che esorbita dalla vicenda d'amore e la supera, nel momento stesso nel quale la ingloba entro una più matura e più vasta e infine definitiva esperienza di vita.

D’altra parte il "canzoniere» amoroso non appare come una parte a sé stante, isolata entro un organismo più vasto: non solo perché plurimi richiami legano una parte all'altra (a ciò sarebbero sufficienti, da sole, le due canzoni «aragonesi» VI e VII, già nel codice e poi in $A$ ), ma perché è d'immediata evidenza, direi, l'unità di tono e di linguaggio e d'atteggiamento che fa dell'Endimione un organismo unico. Abbiamo visto come Cariteo per primo abbia felicemente caratterizzato la sua poesia amorosa come un "canto del cigno», e come proprio in quanto tale il suo canto sia sempre rivolto all'esterno, gridato innanzi al mondo in una dimensione che si può ben definire teatrale la quale, investendo altri interlocutori oltre Luna, assume in maniera ancor più evidente qualcuno di quei caratteri che sin dalla fortunata definizione del D'Ancona si conviene di definire "cortigiani». 31 Onde non sarebbe difficile mostrare come dalla natura "esterna» ed essenzialmente testimoniale di questa poesia dipendano strettamente altri elementi qualificanti, tra loro perfettamente congruenti: per esempio, la predominante affermativa ed esclamativa; la spiccata attitudine canora (ci si ricordi del Cariteo strambottista, maestro di Serafino Aquilano); la notevole semplicità sintattica, che spesso fa coincidere gli enunciati con il verso singolo o con il distico, e s'appoggia a un uso assai intenso delle figure del parallelismo e della ripetizione, in tipica ed evidente funzione strutturante e chiarificante, ecc. Un solo esempio al proposito. Il distico che chiude la penultima stanza della canzone III, Non posso homai tener le fiamme eterne, 65-66, in $A$ suonava: «allora il crederò, quando pur senta / ch'Amor 
possede il cor che mi tormenta», e in $B$ è così mutato: "Allora il crederò, quando veda io, / ch'Amor tenga quel cor che tene il mio». Ecco, in questo tipo di correzione Cariteo appone il suo speciale marchio, che si rivela inconfondibile, per restare all'unità minima del verso, anche in casi come: «et quel che muor non pò morir secreto» (canzone IV, S'alcun conforto al misero è concesso, 48); "per che giungeti foco al foco mio?» (LXXXIII 3); "che per luce di luce io son diviso» (CIV 8); «al gusto dolce, e dolce a la salute» (CLXXXIV 6), e così via. Ancora, la terzina finale del sonetto e il distico a rima baciata che chiude le stanze delle canzoni (ciò avviene in 16 canzoni su 20) hanno spesso il forte rilievo di un'affermazione estrema e sigillano in modo eloquente il motivo svolto nelle quartine, che appare come un caso particolare di una verità assoluta che infine non si può non gridare ai quattro venti. E a ciò perfettamente collabora quella particolare cifra stilistica del Cariteo che consiste nell'ampio uso della formula del gerundio+presente implicato o reversibile, per dir così (vivo morendo / muoio vivendo; morendo canto / muoio cantando $[\ldots]),{ }^{32}$ attraverso il quale lo spazio interamente dato e tutto presente della situazione, amorosa e no, si trasforma in destino. Ma non voglio insistere in analisi che altri ha già fatto benissimo. Semmai, m'importa sottolineare che quello che vale per la prima parte dell'Endimione, quella costituita dalle liriche già in $A$, vale altrettanto là dove il motivo amoroso che dominava nelle liriche della prima raccolta si articola in una vicenda (la partenza di Luna e il rimpianto, e gli anniversari), e lascia poi il posto a cose diverse. Nel canzoniere, infatti, all'amore non s'affianca solo l'ambizione di farsi cantore della dinastia, ma soprattutto prende via via maggiore spazio e finisce per dominare incontrastato uno stretto e affettuoso e rammemorante colloquio con gli amici più cari, scampati al naufragio della dinastia aragonese e tuttavia fedeli alla memoria di quei re sfortunati. E Napoli diventa il luogo magico di un'esperienza conclusa e irripetibile: il luogo della vita stessa, che se n'è fuggita via prima con Luna e poi con gli Aragonesi, inchiodando lui, Cariteo, all'amara dolcezza del ricordo. Non solo a questa, però, dal momento che il sopravvissuto poeta non solo ricorda ma — ed è su ciò che propriamente si fonda il carattere etico della sua poesia, e in ultima analisi il suo fascino - celebra, e celebra perché vuole rivendicare il senso e il valore del proprio passato. Ecco: l'Endimione è un canzoniere che non percorre l'arco di un amore ma di una vita, riproponendone i contenuti forti e l'intatta fedeltà ad essi, ora che tale fedeltà è rimasta sola a riempire l'ultimo spazio che resta, prima della morte. Da questo punto di vista l'amore per Luna e l'amore per i re aragonesi e quello per gli amici e quello per Napoli hanno uno spazio certamente diverso, ma non sono, in essenza, cose diverse rispetto a quella che è, appunto, la struttura profonda del canzoniere, che non è data dai tempi di una o unaltra vicenda, ma piuttosto dalla loro somma che torna a dispiegarsi in un'opera che vuol essere, prima di tutto, un supremo atto di testimonianza. ${ }^{33}$

32. Su questo costrutto, vedi già E. FEnZI, La lingua e lo stile, cit., p. 68 s.; Beatrice BARBIELLINI AMIDEI, Alla Luna, cit., p. 180-181.

33. Onde per parte mia, trovo perfette le parole di Croce, là dove giustamente rifiuta il tono beffardo usato dal D'Ancona, e dice, del Cariteo: «egli sentiva nobilmente e nobilmente 
Così la concepisce, del resto, il Cariteo, che ha piena coscienza del proprio fine. Leggiamo i primi sonetti del canzoniere, quelli che appaiono la prima volta nell'edizione definitiva del 1509 , e il primo di tutti, Se 'l foco del mio casto, alto desio. Polemicamente, Cariteo rivendica la purezza e la nobiltà del suo amore per Luna, del quale non ha affatto da pentirsi come di «insano e folle errore», ma sì da gloriarsene quale espressione la più nobile possibile della sua aspirazione a conquistare «vero honore». La sua dea è stata esempio angelico in terra, e: "Io l'adorai come sustantia pura, / da presso e da lontan: ché l'huom non erra / il fattor adorando in sua fattura». Con tessere petrarchesche (e dantesche) Cariteo costruisce dunque un sonetto introduttivo che dice l'esatto contrario di quello che diceva Petrarca: egli non si pone in alcun modo come "altr'uom da quel ch'i'sono", non condanna il passato in nome della saggezza presente, non si pente di nulla (ancora poco avanti, son. V, Benché la turba errante hor non estime 3: "né dal mio canto in dietro io mi retiro»), e non esibisce infine una sofferenza amorosa che possa essere quanto meno d'esempio e ammonimento agli altri. ${ }^{34}$ Il tempo non l'ha mutato, insomma, ma ha semmai confermato il valore delle sue scelte che possono legittimamente ripresentarsi per quello che furono, promosse, così come furono, senza incrinature, nel pantheon della memoria. Non è questo un termine scelto a caso. Dopo aver esaltato Ferrandino nel sonetto III, Principe, sol di Alcide in terra un pegno, ecco infatti cosa il poeta, sull'evidente traccia di Virgilio, Georg. III 10-16, si propone di costruire, nel IV, che trascrivo per intero:
Ad quanto un cor gentile ama e desia
le mie speranze e voglie hor son sì pronte,
ch'io spero anchor di lauro ornar la fronte
nel dolce luogo dove io nacqui pria.
Primo sarò che 'n l'alta patria mia
condurrò d'Aganippe il vivo fonte,
venerando di Giove il sacro monte,
se morte dal pensier non mi disvia.

esprimeva un momento della storia napoletana e italiana [...]» (Benedetto Croce, Poeti e scrittori del pieno e del tardo Rinascimento, Bari: Laterza, 1945, I, p. 36-43: cit., p. 36). Ma ancora, confutando l'accusa di «spagnolismo-secentista» che proprio il Cariteo avrebbe introdotto, secondo D'ANCONA, nella lirica italiana, vedi La Spagna nella vita italiana, cit., p. 68-69.

34. Non so di esordi simili, così integralmente positivi (ma a Cariteo è particolarmente vicino Serafino Aquilano, I, L'aquila che col sguardo affisa el sole): esalta il proprio amore, ma parla di guerra e di perdita della vita e della libertà Giusto de' Conti, nei primi tre sonetti de $\mathrm{La}$ bella mano, e, per quanto raccomandi ai giovani d'amare, Boiardo dichiara che "quel che alora mi piacque, ora mi dole» (Amorum libri, I, Amor, che me scaldava al suo bel sole 4). Il De Jennaro spera che altri sia ammaestrato dalle sue sofferenze (I, Chi vuol dell'altrui vita amaistrarse), e un atteggiamento oscillante ha pure Lorenzo de' Medici, che nei sonetti proemiali del Canzoniere si augura di essere esempio agli altri, e afferma: «e già mi pento della prima impresa» (III, Già sette volte ha Titan circuito 10). E qualche ombra, anche se leggera, è pure nel Sannazzaro [...] 
E ' $n$ su la riva del purpureo fiume io vo' constituire un aureo templo, in memoria del mio celeste lume.

E tu, Aragonio sol, ch'or io contemplo, sarai del primo altare il primo nume, ché de divinità sei primo exemplo.

In Barcellona, dunque, «l'alta patria mia», sarà egli, Cariteo, a far fluire per primo il sacro fonte delle Muse, ad esso consacrando il «monte di Giove», il Monjuich (Mons Iovis), e lì, lungo le rive del Llobregat (rubricatus, il "purpureo fiume»), egli ancora costruirà un tempio d'oro in memoria di Luna: e nel tempio l'altare principale sarà quello del "primo nume», Ferrante [...] D'essere il primo vero poeta barcellonese, Cariteo lo ribadisce sùbito dopo, nel sonetto $\mathrm{V}$ appena sopra citato, dicendosi sicuro della immortalità che i suoi versi d'amore gli assicureranno e proclamando, in fine: «So che poi del mio fin sarà quieta / l'invidia, che si pasce hor in me vivo, / et havrà Barcellona il suo poeta». ${ }^{35}$ Restiamo, tuttavia, al sonetto IV, e in particolare alla sovrapposizione del mito di Luna e del mito del re. Di re Ferrante, abbiamo detto, seguendo Pèrcopo che annota: «Scritto quando Ferrante I era ancor vivo $(† 25$ gennaio 1494): cfr. v. 12». Ma è proprio così? Sono sicuro di no: ha infatti certamente ragione la Barbiellini Amidei nell'osservare che i sonetti proemiali "furono scritti per ultimi, appositamente per la sistemazione della raccolta definitiva del $1509 » .{ }^{36}$ Non solo perché essi mancano nel codice e nell'edizione del 1506 (questo è semmai un indizio di probabilità, non una prova), ma perché assolvono a una precisa funzione strutturale, corrispondendo con la canzone finale, la XX, al Carafa, in modo tale da «chiudere» l'intero canzoniere entro un univoco schema autoesegetico. Leggiamo la prima strofa della canzone, v. 1-11 (lo schema metrico è quello della canzone 359 di Petrarca, Quando il soave mio fido conforto):

Non temo homai che 'l pelago d'oblio sommerga il mio miglior ne l'onda horrenda; ché nel mondo conven che fulga e splenda, a mal grado d'invidia, il nome mio. Vedrò pur vivo il fin del bel desio: sarà per me quel roseo Rubricato più noto et illustrato;

per mia cagion più celebre anchor fia la prima patria mia, ch'io rigarò di Giove il sacro monte con l'acque eterne del Pïrio fonte.

35. V. 9-14: «Et son secur, che quanto io canto e scrivo / di quel mio chiaro e lucido pianeta / vivrà, quand'io sarò di vita privo. / So che poi del mio fin sarà quieta / l'invidia, che si pasce hor in me vivo; / et havrà Barcellona il suo poeta».

36. Beatrice BARBielli Amidei, Alla Luna, cit., p. 73 e 114 . Ma è diversa l'ottica con la quale la studiosa considera la cosa, e diverso il discorso che ne deriva. 
Tra le altre precise corrispondenze, è da osservare come l'alta patria mia corrisponda ora a la prima patria mia: nel sonetto CLXXII, infatti, scritto nel lasciare Napoli per l'esilio romano, egli saluta la la sua seconda patria, Napoli: «Seconda patria mia, dolce Sirena, / Parthenope gentil [...]», accompagnandola, in fine, con il ricordo della patria prima, Barcellona: «dal giorno che lasciai la patria avita, / io fui da fati iniqui exercitato!». Ma si veda anche l'incipit del sonetto CXCII, al catalano Gerolamo da Colle, scritto nel 1507-1508: "O de la patria mia splendore e gloria, / Hieronimo di Colle [...]», e il sonetto CCXIV, per la morte in Roma di un Filippo barcellonese, 9-10: «Pianga Barcinio, antiqua patria mia / con sua militia e i Consoli honorati $[\ldots] »$. È con il crollo della dinastia aragonese, insomma, e durante l'esilio romano, che Cariteo torna finalmente a rievocare con tanta intensità di sentimento la propria vera patria, quasi che questo esilio l'abbia messo dinanzi al fatto che tutta la sua vita era stata, in qualche modo, un lungo esilio, sin dal momento in cui aveva abbandonato una patria che diventa, ora, là, sull'estremo lembo della vita, il simbolo di un neoplatonico ritorno alla perfezione e alla purezza originarie. Ed è allora, a partire dall'esilio romano, appunto, che la memoria delle proprie lontane radici lo risarcisce di tante perdite, e che in essa egli ricompone un intero percorso di vita: dalla fanciullezza che proprio là, in Barcellona, aveva ricevuto il dono della poesia, alla maturità napoletana devota all'amore e alla dinastia regnante, e infine alla presente certezza della propria gloria immortale che la sua stessa fedeltà gli ha garantito. Basti per ciò l'importante sonetto, scritto, di nuovo, negli anni romani e diretto ad Agostino Chigi, CCXXXIX, nel quale va anche osservato come, contestualmente, Cariteo rovesci il tradizionale topos del poeta che assicura la fama imperitura agli uomini di governo. Non è così: sono infatti i re che accolgono nel fulgore della loro gloria e illuminano l'opera del poeta, che solo ad essi deve la sua speranza d'immortalità (mio il corsivo):

Augustin mio, non creder che soggetto

a morte in tutto io sia: quand'io fui nato

presso il sonante roseo Rubricato,

mi nutrì de le Muse il latteo petto.

Napol mi tenne poi nel bel ricetto

sette lustri, invaghito, inamorato

del suo dolcior divino: ivi pregiato

fu 'l canto mio di Re d'alto intelletto.

Fulgon nei versi miei lor nomi, ond'io

spero tal parte aver di lor fulgore

che sarà sempiterno il viver mio.

[...]

Ma si veda ancora, infine, in che modo Cariteo ridisponga ancora una volta gli elementi portanti (metto in corsivo quelli già visti) del suo mito nell'ultima 
opera, la Pascha, il poemetto in sei canti d'argomento prima religioso e poi storico, ${ }^{37}$ I $37-48$ :

\author{
O quando fia quel dì, Muse benigne, \\ che 'n la mia patria prima io vi conduca, \\ in quelle alte magion di gloria digne? \\ Là conven che 'l mio nome splenda e luca, \\ rimembrando l'onor ch'al cielo extolle \\ il mio bel Sannazar, maestro e duca; \\ il suo Sebeto e 'l bipartito colle \\ Vesuvio, e i lauri ch'adornaro il ciglio \\ del Re che 'l cielo innanzi tempo volle. \\ Sotto 'I monte di Giove, in sul vermiglio \\ fiume, poner io spero un templo d'oro \\ a la madre del ciel, figlia del figlio.
}

37. Come ho accennato, si tratta di un poemetto in terza rima, in sei cantici, composto in grave età (I 4), e certo dopo la morte del Pontano, nel settembre 1503: I 54). Nel primo, dopo un'invocazione a Dante e Petrarca e dopo aver espresso il desiderio di cantare i "pascali, immensi, alteri onori» in gara con il De partu Virginis del Sannazzaro, Cariteo comincia seguendo passo passo la narrazione evangelica, in ispecie quella di Giovanni, sino alla discesa di Cristo al Limbo, l'ascesa delle anime liberate al cielo, dove Davide intona un inno di ringraziamento. Nel secondo, seguendo Luca, tratta delle apparizioni di Cristo risorto alla madre, a Maddalena e ai due pellegrini che si recano a Emmaus (uno dei due si chiamava Cleofa), che poi Dio accoglie in Paradiso. Nel cantico terzo, c'è una raffigurazione allegorica della Pace, e si esalta poi la Giustizia, la Sapienza e l'Amore. Ad essa segue l'apparizione di Cristo agli apostoli (III 67 s.) e la sua ascesa al cielo, dove le anime degli innocenti fatti uccidere da Erode intonano un inno di lode e preghiera. Il quarto si apre con una invocazione alla Beatrice dantesca, e prosegue con Cristo che fa portare la Vergine in cielo e la fa incoronare (IV 61 s.). Si passa poi all'esaltazione dei re Magi (qui è inserito anche un ricordo di Ferrandino, v. 124-132), pur essi levati al Paradiso. Il quinto, esalta ancora la sapienza e la fede dei Magi che raccomandano a Dio la loro discendenza: ed è questa discendenza che è ampiamente illustrata da Cloto, sino a quasi tutto il cantico VI, riprendendo l'antica credenza che voleva che la famiglia Del Balzo (De Baux), trapiantata dalla Francia in Italia nel 1265 con Bertrando gran giustiziere di Carlo I d'Angiò, discendesse da Baldassarre, quello dei Magi che per primo vide la stella che li guidò a Betlemme. Ma Cloto fa prima una rapida rassegna (con la quale il cantico termina) dei re aragonesi, da Alfonso il Magnanimo a don Federigo e al figlio di costui, Ferrante, che Cariteo ancora spera di vedere un giorno sul trono di Napoli. Il cantico VI, come s'è detto, è occupato dalle genealogie che vedono intrecciate le discendenze di quattro fratelli (per parte di madre), Inico e Alfonso D'Avalos, e Inico e Ferrante di Guevara, venuti in Italia al seguito di Alfonso il Magnanimo; di Tristano di Chiaromonte imparentato con i Del Balzo attraverso la moglie, e di Bertrando Del Balzo. In particolare, Cariteo esalta l'unione di questa famiglia con gli aragonesi, con Isabella prima moglie di Ferrante I, e con Isabella moglie di don Federigo, alla quale Cariteo già aveva dedicato i versi forse più belli della Metamorfosi (II 58-72). Aggiungo che, di là da affrettate stroncature, l'opera meriterebbe maggior attenzione, come del resto le stesse Metamorfosi, e che ha perfettamente ragione a richiamare a ciò Isabel SEGARRA AÑON, in una lunga e bella recensione al volume di Giovanni Parenti, «A propòsit d'una lectura sobre Benet Garret "Il Cariteo" (1450-1514)» Faventia, 20/1, 1998, p. 85-94 (vedi in part. p. 90-92). 
Non credo occorra insistere, a questo punto, sul fatto che il sonetto IV, dal quale ho preso le mosse, Cariteo l'abbia scritto proprio in questa ultima fase della sua poesia e della sua vita, quando egli dà corpo, in maniera così suggestiva, all'estremo sogno del ritorno in patria. Tanto forte e importante, questo sogno, che azzardo un piccolo sospetto che non riesco a togliermi di mente, e cioè che il poeta, vicino alla fine, abbia realmente progettato di tornare a Barcellona, per morirvi (del resto, della sua morte e della sua sepoltura in Napoli non è rimasta traccia). Come che sia, il sogno diventa possibile perché tutto il mondo che sta alle sue spalle è irrevocabilmente perduto: lo è Luna e lo sono i suoi re, ma lo sono anche Posillipo e il Sebeto e Mergellina, e insomma quella Napoli ch'è ormai solo un luogo dell'anima e che è così amorevolmente e affettuosamente ricordata nelle ultime terzine della Pascha, VI 169-181:

So ben che trovarete assai più degno sacerdote di voi, Pierie dive,

ma non più pio né più fidele ingegno.

Questo cantava a i lauri a l'aure estive

tra 'I mio Summontio, Pardo e Galateo,

anime eternamente al mondo vive,

quando di quel liquor partenopeo

Sincero mi pascea, dolce cantando

con le Carìte ond'io fui Cariteo,

di poi che quel secondo almo Ferrando,

sepolto in terra il bel corporeo velo,

suoi secreti pensieri in me lasciando

con penne di pietà volò nel cielo.

Tornare a Barcellona, alla patria prima, e porre lì il tempio d'oro della propria opera, consacrata prima a Luna e poi ai re d'Aragona e infine alla Vergine, significa allora chiudere il cerchio e dare un senso compiuto al gran viaggio della propria vita e della propria poesia. Non si tratta quindi di un motivo tra altri, ma propriamente del grande mito poetico attraverso il quale Cariteo osa sottrarsi al tempo e alle sue rovine, per vivere nell'eternità garantitagli dal monumento aere perennius da lui costruito. ${ }^{38} \mathrm{E}$ si comprende bene, allora, come avvenga che tutto ciò che sta positivamente entro il perimetro del monumento sia appunto «monumentalizzato» o, a dir propriamente, sacralizzato. E come questa tensione sacralizzante s'appoggi a una forte funzione tra il rituale e il rievocativo della parola poetica che investe, per non portare che un visibilissimo esempio, i nomi. Si osservi infatti la forte carica evocativa e sentimentale che i nomi hanno, in questa tarda stagione di Cariteo: i nomi dei re, i nomi delle grandi famiglie e delle loro memorande genealogie, i nomi

38. Per questo motivo oraziano, con particolare riferimento alla citata canzone $\mathrm{XX}$ al Carafa, vedi Irene Segarra AÑon, El tema del monumentum horaciano en Benet Garret, "il Cariteo", poeta y humanista catalán en la corte catalano-aragonesa de Nápoles, nel vol. miscellaneo Antonio De Nebrija: Edad Media y Renacimiento, Salamanca: Ediciones Universidad de Salamanca, 1994, p. 505-512. 
dei poeti e degli amici, i nomi dei luoghi [...] Raramente Cariteo usa le perifrasi. I nomi dei destinatari dei suoi sonetti sono sempre pronunziati in tono alto, pieno: e anche i nomi più semplici, quelli degli amici spesso accompagnati dal possessivo mio, cessano d'essere tali, e diventano emblemi, segni di valore. Meriterebbe fermarsi su questo aspetto della poesia di Cariteo: ${ }^{39}$ io non saprei ricordare tanti altri poeti con altrettanta forza sappiano chiamare i nomi alla poesia: che sappiano "fare poesia» con i nomi, caricati di vibrazioni così intense. Tutta l'ultima parte dell'Endimione è piena di nomi, e di tali nomi vive. Qualche esempio lo si può ricavare dai versi già citati: qui, per finire, mi limito ad aggiungerne un altro, costituito dal bel sonetto CCXXXVIII a Michele Dolce:
Michele, a cui le Muse il dolce accento, la dolce lira diero e 'l dolce canto, onde tra' più soavi il pregio e 'l vanto e di Dolce acquistasti il cognomento, deh! non ti consumar sempre in lamento. Le lagrime abondanti affrena alquanto, ché ritornar non può per molto pianto colui che ti fu rapto in un momento. Rende 'l tuo canto a la bella marina

39. Sarò probabilmente vittima della mia spiccata simpatia per Cariteo (alla fine, potrei semplicemente dire che è un poeta che mi piace), ma trovo che anche nelle più banali e ripetitive formule di omaggio egli sappia sempre scandire i nomi con abilità e forza. In posizione incipitaria, per esempio, come in CCV 1: "Magnanimo Scipion Philomarino», o in CXCIII 1-2: «L’excelsa tua vertù, grande Almirante, / conte Villamarino [...]»; CXCVII 1: «Degno di tuoi magior, gentil Camillo»; CCCIII 1: «De la Monica sterpe eximio honore, / Ferrando [...]", e in contesti più ampi, come in CC 9-11: "Quella contessa candida, Vittoria, / che li Cantelmi exalta in alta sella, / ed è di Bautii heroi non poca gloria», o fuori dall'Endimione, nella Metamorfosi, II 130-134: «Del qual fulgor partìcipi fai quelle / anime grandi d'Avelo e d'Aquino, / chiare per lor vertù, per te più bella: / lo strenuo Roderico e 'l bel Martino / con Hyppolita dea, che più risplende», ecc. Certo è nei luoghi di Napoli, e negli amici, che risuona più effusiva la vena sentimentale e affettuosa, della quale abbiamo già visto alcuni belli esempi: ma ancora CLXXIX 1-4: "Dove mezzo hor son io, sacre Sirene, / con voi, volesse il ciel! vi fussi intero, / et udissi il cantar del mio Syncero / nel Mergillino suo, dolce Hyppocrene!», o in questo bel distico della Metamorfosi, I 22-23: «Era a veder in quella prospettiva / Napol superba, e 'l bel Vesuvio monte», e ancora nella Pascha, I 5254: «Tra tanto hor qui, nel bosco Antinïano, / tra gli odorati lauri e i bei myrteti, / suscitarem Vergilio e 'I gran Pontano". Le citazioni sarebbero molte, in ogni caso, ma voglio qui almeno accennare al "gioco sul nome», che Cariteo impiega con frequenza (come nel sonetto a Michele Dolce, citato poco avanti nel testo), nell'intero sonetto al cardinale Oliviero carafa, per esempio, CLIX, Frondosa arbor, gentil, sempre florente; in CLXXIX ad Angelo Colocci, 1-2: "Colotio, di vertù vero cultore, / degno del nome angelico e divino»; in CLXXXVI 9-11, al cardinale Giuliano Cesarini (ovviamente, il gioco è su Cesare); in CCI 6, a Consalvo Fernando de Heredia: "Tu sei de la vertute Heredia herede»; nella canzone XX 99, a Vincenzo Carafa: «Vincenzo, vincitor giamai non vinto», ecc. Tutto ciò, di là dal giudizio che si vorrà dare e fatta l'abbondante tara delle convenzioni encomiastiche, resta significativo, a mio parere, dello speciale valore che Cariteo annette al nome, e alle risonanze poetiche e mentali di cui è portatore. 
de le Sirene albergo ameno e lieto, templo sacrato a la Musa divina. Pausillipo t'invita e 'l tuo Sebeto, la Platamonia fresca e Mergillina, sotto odorati citri e 'l bel laureto.

Napoli con i suoi re, insomma, è, nella memoria, la sicura e calda "cellula di miele» che l'aveva accolto e protetto e aveva dato un senso a tutto il suo lavoro; Barcellona, la "prima» patria, gli si ripresenta da chissà quale lontananza come il miraggio della salvezza, fuori dal tempo, di quel senso, ora che il duro presente l'aveva impietosamente distrutto. Intanto proprio qui, in questo estremo assediato riparo della Napoli spagnola, il Sannazaro lavorava al poema latino sulla natività, il Summonte curava la pubblicazione delle opere del Pontano, morto nel 1503, e Cariteo attendeva al poema sulla resurrezione, versificando in un colorito linguaggio biblico e latineggiante i testi evangelici, e tornando a ripopolare i suoi versi di tutti gli attori della passata stagione aragonese. E quello che a lui, Cariteo, era rimasto di più solido non era infine un intervento di segno positivo nelle vicende del mondo, ma la somma dei suoi versi e una lunga esperienza morale e poetica. In questa luce, approfittando della stampa di tutta la sua opera, nel 1509, era importante segnare bene l'articolazione interna dei motivi che avevano portato a superare l'esperienza amorosa, ma era soprattutto importante ribadire la ferma fedeltà al proprio mondo, a cominciare dal giovanile amore per Luna, come mostrano i sonetti proemiali aggiunti al primitivo corpus di $A$. Sì che par giusto concludere che l'Endimione intende essere "tutto» Cariteo, nel senso che tutto quello che contiene è leggibile seguendo il filo che passa per la sua vita e lega i momenti in cui il poeta guardò, sempre con molta serietà, a se stesso e al suo lavoro. Quale sia questo filo, non è difficile trovarlo, appunto, nella fedeltà a una morale e a un'estetica in senso alto "cortigiana", che poneva al proprio centro una nozione pervasiva di regalità, ed esaltava e moltiplicava gli echi più sonori della parola poetica. E, armato di questa fedeltà, Cariteo è riuscito davvero a salvare e a sublimare la propria esperienza di uomo e di poeta. 